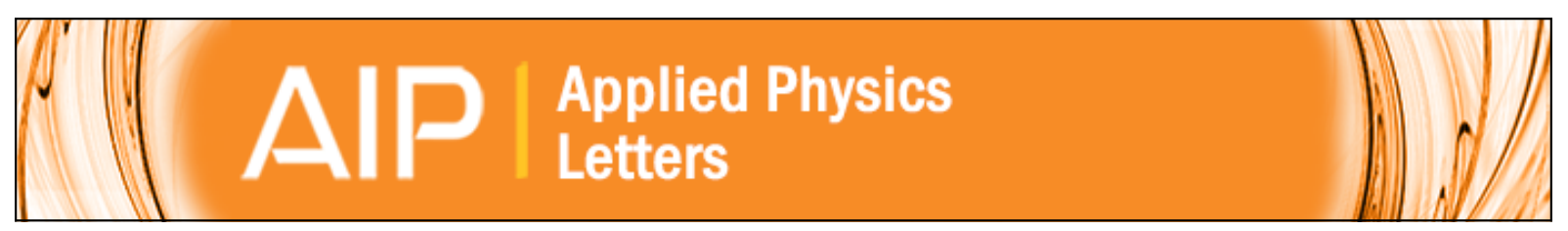

\title{
Plasmonic light yield enhancement of a liquid scintillator
}

Lindsey J. Bignell, Eskender Mume, Timothy W. Jackson, and George P. Lee

Citation: Applied Physics Letters 102, 211902 (2013); doi: 10.1063/1.4807146

View online: $h t t p: / / d x . d o i . o r g / 10.1063 / 1.4807146$

View Table of Contents: http://scitation.aip.org/content/aip/journal/apl/102/21 ?ver=pdfcov

Published by the AIP Publishing

\section{Advertisement:}

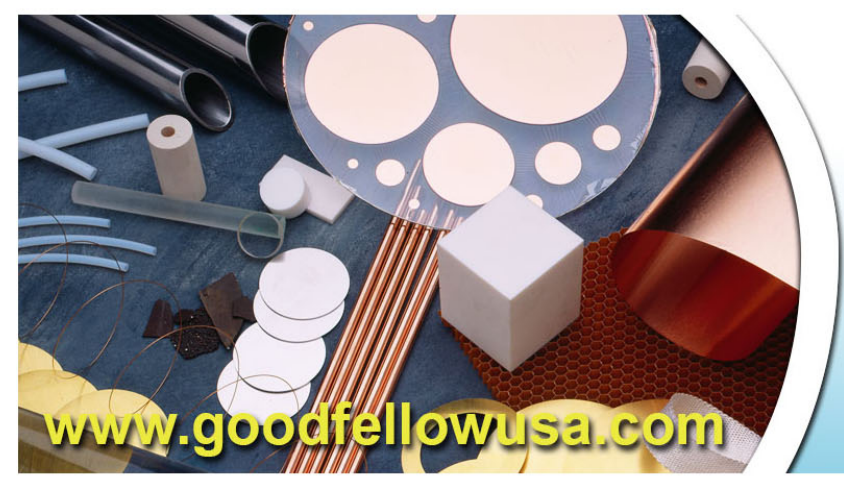

BOOdFEl/OII metals • ceramics $\cdot$ polymers composites $\bullet$ compounds • glasses Save $5 \%$ B Buy online 70,000 products $\cdot$ Fast shipping 


\title{
Plasmonic light yield enhancement of a liquid scintillator
}

\author{
Lindsey J. Bignell, ${ }^{1, a)}$ Eskender Mume, ${ }^{1,2}$ Timothy W. Jackson, ${ }^{1}$ and George P. Lee ${ }^{3}$ \\ ${ }^{1}$ Australian Nuclear Science and Technology Organisation, Lucas Heights, New South Wales 2234, Australia \\ ${ }^{2}$ Center of Excellence in Anti-matter Matter Studies, Research School of Physics and Engineering, \\ The Australian National University, Canberra, Australia \\ ${ }^{3}$ Department of Materials Engineering, Monash University, Melbourne, Australia
}

(Received 3 April 2013; accepted 2 May 2013; published online 28 May 2013)

\begin{abstract}
We demonstrate modifications to the light yield properties of an organic liquid scintillator due to the localization of the tertiary fluorophore component to the surface of Ag-core silica-shell nanoparticles. We attribute this enhancement to the near-field interaction of Ag nanoparticle plasmons with these fluor molecules. The scintillation light yield enhancement is shown to be equal to the fluorescence enhancement within measurement uncertainties. With a suitable choice of plasmon energy and scintillation fluor, this effect may be used to engineer scintillators with enhanced light yields for radiation detection applications. (C) 2013 AIP Publishing LLC. [http://dx.doi.org/10.1063/1.4807146]
\end{abstract}

The demand for brighter, cheaper, and faster scintillators for applications such as border screening detectors ${ }^{1}$ and nuclear medicine imaging ${ }^{2}$ has seen recent innovation in scintillation materials research. This has included the development of inorganic scintillating crystals, ${ }^{3,4}$ organic scintillators, ${ }^{5-7}$ inorganic/organic hybrid materials, ${ }^{8}$ and scintillators incorporating nanomaterials. ${ }^{9-11}$ However, despite some recent theoretical advancements, ${ }^{12,13}$ the properties of scintillation materials cannot be accurately predicted a priori, hindering the design of scintillators with desirable properties.

Recent advances in fluorescence enhancement provide an avenue for engineering scintillation materials. Plasmonic nanoparticles (NPs) induce enhanced light yields and reduced decay times in nearby fluorophores. ${ }^{14,15}$ This extraordinary plasmonic enhancement is due to the large optical absorption cross section for the excitation of the nanoparticle's Localized Surface Plasmon Resonance (LSPR), which in turn generates extremely high $\vec{E}$ fields near the surface of the nanoparticle. Resonant dipole-dipole coupling between the LSPR $\vec{E}$ field and nearby fluorophores can be achieved, provided there is a suitable molecular transition, allowing efficient excitation of the fluorophore. Plasmonically enhanced fluorescence has potential application in light-emitting diode (LED) ${ }^{16,17}$ and biomolecular sensing ${ }^{18}$ technologies, amongst others.

By incorporating appropriate plasmonic nanoparticles into scintillation materials, an analogous enhancement can be observed. In this Letter, we report the plasmonic enhancement of the light yield of a liquid scintillator.

The excitation mechanism associated with scintillation is more complicated than fluorescence. Rather than direct optical excitation of the fluorophore, scintillation requires excitation transfer from the solvent to fluorophore, and optionally additional excitation transfer stages to secondary and tertiary wavelength-shifting fluorophores. For this study, we have designed a liquid scintillator with 9-Aminoacridine

\footnotetext{
${ }^{\text {a) }}$ Author to whom correspondence should be addressed. Electronic mail: lindsey.bignell@ansto.gov.au
}

(9-AA) covalently bound to the surface of a Ag-core silicashell nanoparticle acting as a plasmonically enhanced wavelength-shifting component (Figure 1).

Scintillation detection does not rely upon the direct excitation of fluorophores by ionising radiation, due to the low fluorophore concentrations that are used to avoid concentration quenching. Rather, solvent excitation is passed either radiatively or non-radiatively via Förster Resonant Energy Transfer (FRET) to nearby fluor molecules. Secondary and tertiary fluor molecules may be excited in a similar manner by the primary or secondary fluorophore, respectively. The nanoparticle-9-AA composite forms a tertiary fluor component in our scintillator and is therefore excited via either FRET or optical excitation by secondary fluorophore emission. The components of the scintillation cocktail were chosen so the emission and absorption spectra of molecules in the excitation chain overlapped sufficiently to allow these optical processes to occur (Figure 2).

A stock scintillator containing $6.6 \mathrm{~g} / \mathrm{L}$ 2,5-diphenyloxazole (PPO) and $0.66 \mathrm{~g} / \mathrm{L}$ 1-4,bis-2 -(5-Phenyloxazolyl)benzene (POPOP) in toluene was prepared and diluted by a factor of 2 using Triton X-100 to allow incorporation of the aqueous phase.

We then covalently bound the 9-AA fluorophore component to the surface of silver-core silica-shell NPs using a cyanate functionalization method. ${ }^{19,20} 10 \mathrm{~g} / \mathrm{L}$ of polyvinylpyrrolidone-stabilized NPs with $10 \mathrm{~nm}$ core radius and $10 \mathrm{~nm}$ shell thickness were dissolved in water. The solution $\mathrm{pH}$ was adjusted to 10.5 using $1 \mathrm{M} \mathrm{NaOH}$. The nanoparticle surface was functionalized with cyanate by adding $7.5 \mathrm{~g} / \mathrm{L}$ cyanogen bromide and tumbling for $60 \mathrm{~min}$. The solution was then diluted by a factor of 2 with a sodium borate buffer, $\mathrm{pH}$ 8.8. Several aliquots were taken and 9-AA was added to make $0.129,0.0656,0.0331,0.0166$, and $0.00833 \mathrm{~g} / \mathrm{L}$ solutions. These were tumbled overnight to allow the 9-AA to covalently bind to the cyanatefunctionalized nanoparticles. Controls were also prepared using an identical process except for the addition of cyanogen bromide, resulting in free 9-AA and non-functionalized NPs of identical concentration to the samples. 


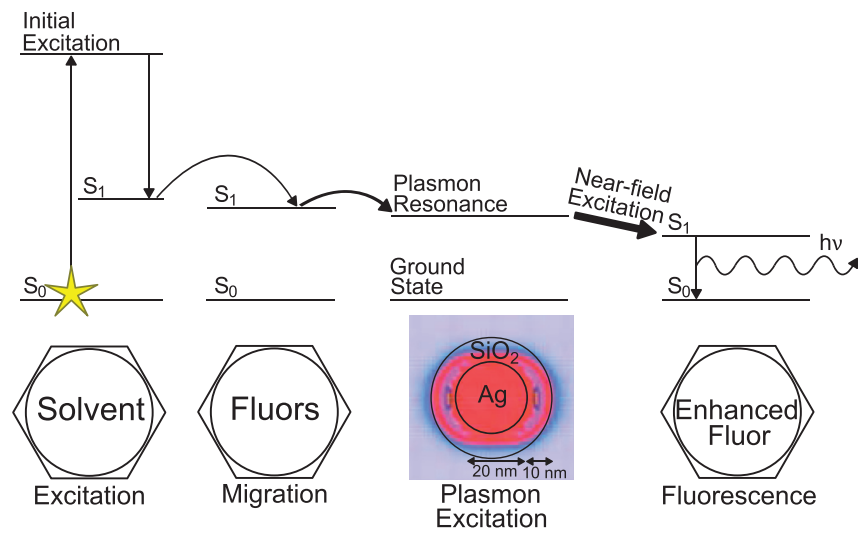

FIG. 1. Schematic of the plasmon-enhanced scintillation process. Ionising radiation deposits excite the solvent, and the excitation migrates to the primary and secondary fluorophores. The large optical absorption cross section of the plasmonic nanoparticle ensures efficient excitation of the LSPR. The surface-bound fluorophore is resonantly coupled to the LSPR near-field, providing enhanced excitation.

$1 \mathrm{~mL}$ of the $0.129 \mathrm{~g} / \mathrm{L}$ 9-AA sample and control were added to separate aliquots with $9 \mathrm{~mL}$ of water. Fluorescence spectra (Figure 3) were taken using a custom-built spectrometer with a $370 \mathrm{~nm}$ excitation source. A fluorescence yield enhancement of 1.23 relative to the control was observed. Given that the fluorescence quantum yield of 9-AA is already close to $1,{ }^{21}$ most of the observed enhancement must be due to the enhanced absorption by the NPs.

Liquid scintillation cocktails were prepared by adding $0.1 \mathrm{~mL}$ of each of the NP-9-AA sample and control solutions to $9.9 \mathrm{~mL}$ of the stock scintillator. Fluorescence spectroscopy of the aliquots with the highest 9-AA concentration was carried out. The fluorescence light yield enhancement is smaller for the scintillation cocktail, at 1.13. This is because POPOP, 9-AA, and the NPs each absorb at $370 \mathrm{~nm}$ (Figure 2), so that competition with the unenhanced POPOP fluorescence component causes an apparent reduction of the enhancement. This additional light yield component may be seen from a comparison of the emission spectra of the aqueous sample and the scintillation cocktail sample (Figure 4). Nonetheless, the observed light yield enhancements relative to the free nanoparticle control give confirmation that the localization

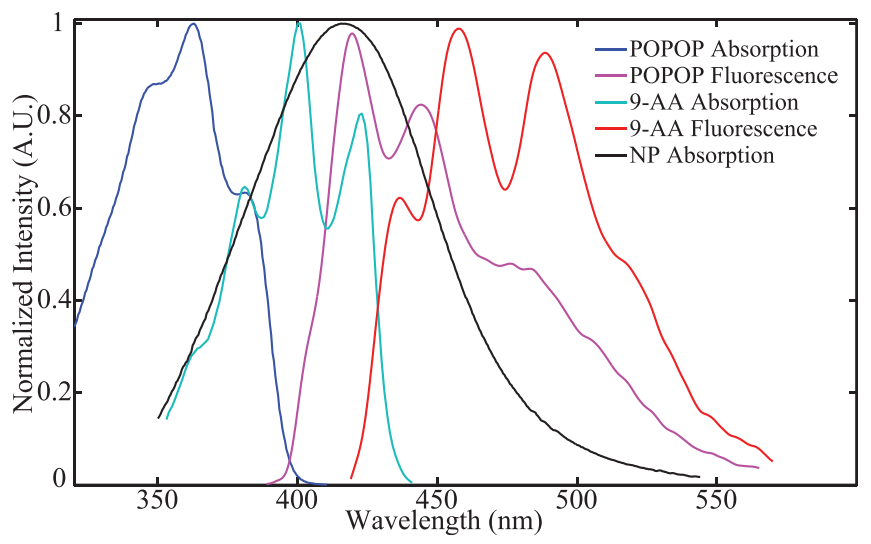

FIG. 2. The absorption and fluorescence spectra for several scintillation components. The secondary fluorophore POPOP emission spectrum is suitable for exciting the Ag-core, silica-shell NP. The 9-AA absorption spectrum allows resonant coupling to the near-field of the excited NPs. The NP absorption spectrum is narrow enough to not attenuate the 9-AA emission spectrum at longer wavelengths.

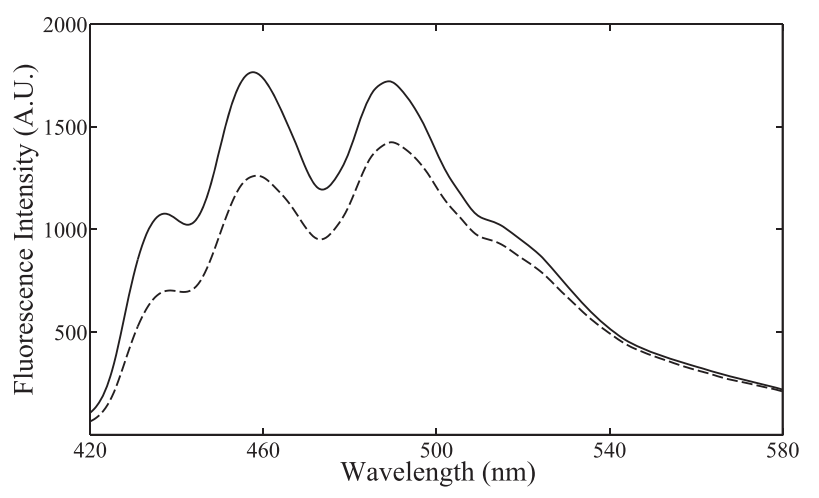

FIG. 3. The fluorescence emission intensity of plasmonic nanoparticle surfacebound 9-AA (solid trace) relative to a free 9-AA control (dashed trace).

of the 9-AA to the near-field of the plasmonic nanoparticles induces resonant plasmon-fluorophore excitation transfer.

In order to assess the scintillation properties of the NP sample and the non-functionalized NP control; each were measured in a custom-built scintillation detector, ${ }^{22}$ with three photodetectors operating in coincidence. $59.45 \mathrm{keV}$ $\gamma$-rays from an external collimated ${ }^{241} \mathrm{Am}$ source were used to excite the scintillator. A comparison of the triplecoincident count rate for these samples indicates a scintillation count rate enhancement of 3.9\%-7\% that varies with the 9-AA concentration (Figure 5). As the only difference between the sample and control solutions is the localization of the 9-AA to the surface of the NPs, we attribute this difference to plasmonic enhancement.

The observed plasmonic scintillation enhancement was not as large as that observed for the fluorescence of the same samples. This discrepancy can be attributed to the differences between the detection techniques. Whereas fluorescence measures the intensity of light emitted by a sample under constant excitation over an integration period, scintillation counting is a triggered measurement of much lower light levels excited by discrete events.

A model relating the scintillation detection efficiency to the light yield can be used to compare these measurements via comparison of the fluorescence and scintillation light yield enhancements. It can be shown ${ }^{23,24}$ that for a spectrum of energy deposits within the scintillator, $S(E)$, that spans the range $\left[0, E_{\max }\right]$, the triple-coincidence detection efficiency may be written as

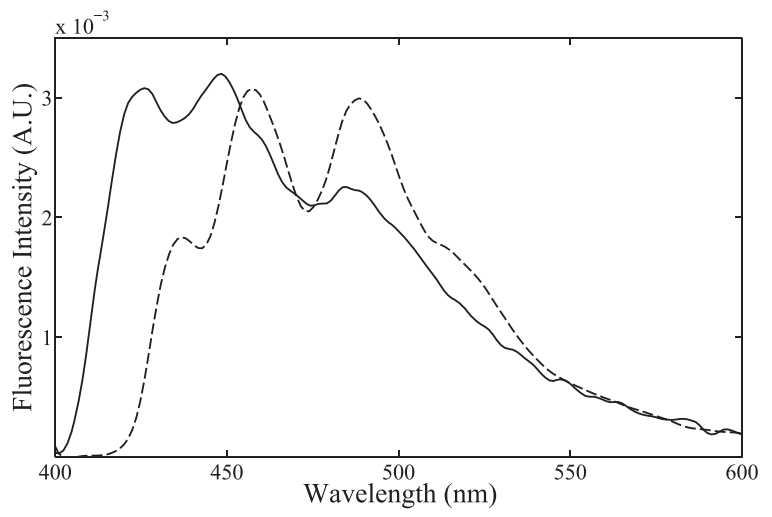

FIG. 4. The normalized fluorescence spectra associated with the 9-aminoacridine-coated plasmonic nanoparticles in water (dashed trace) and emulsified in the scintillation cocktail (solid trace). 


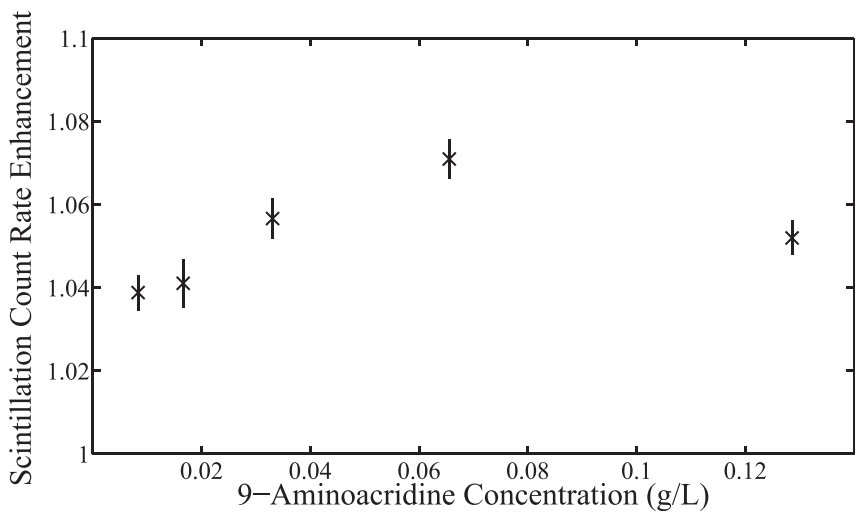

FIG. 5. The scintillation count rate enhancement of 9-AA coated plasmonic nanoparticles relative to a free 9-AA and nanoparticle control, as a function of 9-AA concentration during fabrication. An external ${ }^{241} \mathrm{Am}$ source was used for excitation.

$$
\epsilon_{T}=\int_{0}^{E_{\max }} S(E) \prod_{i=1}^{3}\left(1-e^{-\lambda_{i} E Q(E)}\right) d E
$$

where $\lambda_{i}$ is the mean number of detectable photons incident upon the $i$ th photomultiplier per unit energy deposit in the scintillator and is thus proportional to the scintillation light yield. $Q(E)$ is a correction for the scintillation nonlinearity-for which we have used the Birks Model $^{25}$ with a non-linearity parameter of $k B=0.010 \mathrm{~cm} / \mathrm{MeV}$. We have calculated $S(E)$ for our detector geometry using the GEANT4 Monte Carlo-based radiation transport modeling toolkit, ${ }^{26}$ version $9.5 \mathrm{p} 01$.

We used the Triple-to-Double Coincidence Ratio (TDCR) method $^{27}$ to determine $\lambda_{i}$ for each photomultiplier and calculated the detection efficiency to the external source using Eq. (1).

Using this model, we have calculated that if the scintillation light yield enhancement is equal to the observed fluorescence light yield enhancement of 1.13, the predicted detection efficiency enhancement of the highest concentration 9-AA source is 1.048. This predicted value agrees with the measured value of $1.051 \pm 0.004$ within one standard deviation, confirming that the scintillation light yield enhancement is equal to the fluorescence enhancement.

In summary, we have used 9-aminoacridine covalently bound to colloidal silver-core silica-shell nanoparticles to demonstrate the plasmonic enhancement of the light yield of a scintillator. We have also shown that despite differences in excitation mechanism, the plasmonic fluorescence enhancement is equal to the scintillation enhancement. This suggests that it may be possible to incorporate materials previously developed for fluorescence into plasmonically enhanced scintillators.

These results are expected to be applicable to other scintillation matrices. Naturally amenable materials include plastic scintillators, scintillating paints, and other 2D scintillating surfaces, quantum dot scintillators and scintillating fibres.

The authors thank Dr. Ian Watson for the use of his laboratory facility.
${ }^{1}$ E. R. Siciliano, J. H. Ely, R. T. Kouzes, B. D. Milbrath, J. E. Schweppe, and D. C. Stromswold, Nucl. Instrum. Methods Phys. Res. A 550, 647 (2005).

${ }^{2}$ M. Conti, L. Eriksson, H. Rothfuss, and C. L. Melcher, IEEE Trans. Nucl. Sci. 56, 926 (2009).

${ }^{3}$ K. W. Krämer, P. Dorenbos, H. U. Güdel, and C. W. E. Van Eijk, J. Mater. Chem. 16, 2773 (2006).

${ }^{4}$ N. J. Cherepy, G. Hull, A. D. Drobshoff, S. A. Payne, E. van Loef, C. M. Wilson, K. S. Shah, U. N. Roy, A. Burger, L. A. Boatner, W.-S. Choong, and W. W. Moses, Appl. Phys. Lett. 92, 083508 (2008).

${ }^{5}$ A. Tarancon, J. Garcia, and G. Rauret, Anal. Chim. Acta 463, 125 (2002).

${ }^{6}$ E. V. van Loef, J. Glodo, U. Shirwadkar, N. Zaitseva, and K. S. Shah, Nucl. Instrum. Methods Phys. Res. A 652, 424 (2011) [Symposium on Radiation Measurements and Applications (SORMA) XII, 2010].

${ }^{7}$ N. Zaitseva, B. L. Rupert, I. Pawelczak, A. Glenn, H. P. Martinez, L. Carman, M. Faust, N. Cherepy, and S. Payne, Nucl. Instrum. Methods Phys. Res. A 668, 88 (2012).

${ }^{8}$ A. Yoshikawa, T. Yanagida, Y. Yokota, K. Kamada, Y. Usuki, and M. Nikl, in IEEE Nuclear Science Symposium Conference Record (NSS/MIC) (2009), p. 1524.

${ }^{9}$ S. Saengkerdsub, H.-J. Im, C. Willis, and S. Dai, J. Mater. Chem. 14, 1207 (2004).

${ }^{10}$ S. E. Letant and T. F. Wang, Nano Lett. 6, 2877 (2006).

${ }^{11}$ B. K. Wagner, Z. Kang, J. Nadler, R. Rosson, and B. Kahn, SPIE Proc. 8373, 83730K (2012).

${ }^{12}$ S. Kerisit, K. Rosso, B. Cannon, F. Gao, and X. YuLong, J. Appl. Phys. 105, 114915 (2009).

${ }^{13}$ S. Payne, W. Moses, S. Sheets, L. Ahle, N. Cherepy, B. Sturm, S. Dazeley, G. Bizarri, and W.-S. Choong, IEEE Trans. Nucl. Sci. 58, 3392 (2011).

${ }^{14}$ P. Anger, P. Bharadwaj, and L. Novotny, Phys. Rev. Lett. 96, 113002 (2006).

${ }^{15}$ S. Kühn, U. Håkanson, L. Rogobete, and V. Sandoghdar, Phys. Rev. Lett. 97, 017402 (2006).

${ }^{16}$ K. Okamoto, I. Niki, A. Shvartser, Y. Narukawa, T. Mukai, and A. Scherer, Nature Mater. 3, 601 (2004).

${ }^{17}$ K. Catchpole and S. Pillai, J. Lumin. 121, 315 (2006).

${ }^{18}$ L. B. Sagle, L. K. Ruvuna, J. A. Ruemmele, and R. P. Van Duyne, Nanomedicine 6, 1447 (2011).

${ }^{19}$ R. Axén, J. Porath, and S. Ernback, Nature 214, 1302 (1967).

${ }^{20}$ G. T. Hermanson, A. K. Mallia, and P. K. Smith, Immobilized Affinity Ligand Techniques (Academic Press, New York, NY, 1992), pp. 53-56.

${ }^{21}$ Y. Kubota and Y. Fujisaki, Bull. Chem. Soc. Jpn. 50, 297 (1977).

${ }^{22}$ T. Steele, L. Mo, L. Bignell, M. Smith, and D. Alexiev, Nucl. Instrum. Methods Phys. Res. A 609, 217 (2009).

${ }^{23}$ R. Broda, K. Pochwalski, and T. Radoszewski, Appl. Radiat. Isot. 39, 159 (1988).

${ }^{24}$ A. G. Malonda and B. M. Coursey, Appl. Radiat. Isot. 39, 1191 (1988).

${ }^{25}$ J. B. Birks, The Theory and Practice of Liquid Scintillation Counting (Pergamon Press, Oxford, UK, 1964).

${ }^{26}$ S. Agostinelli, J. Allison, K. Amako, J. Apostolakis, H. Araujo, P. Arce, M. Asai, D. Axen, S. Banerjee, G. Barrand, F. Behner, L. Bellagamba, J. Boudreau, L. Broglia, A. Brunengo, H. Burkhardt, S. Chauvie, J. Chuma, R. Chytracek, G. Cooperman, G. Cosmo, P. Degtyarenko, A. Dell'Acqua, G. Depaola, D. Dietrich, R. Enami, A. Feliciello, C. Ferguson, H. Fesefeldt, G. Folger, F. Foppiano, A. Forti, S. Garelli, S. Giani, R. Giannitrapani, D. Gibin, J. J. Gomez Cadenas, I. Gonzalez, G. Gracia Abril, G. Greeniaus, W. Greiner, V. Grichine, A. Grossheim, S. Guatelli, P. Gumplinger, R. Hamatsu, K. Hashimoto, H. Hasui, A. Heikkinen, A. Howard, V. Ivanchenko, A. Johnson, F. W. Jones, J. Kallenbach, N. Kanaya, M. Kawabata, Y. Kawabata, M. Kawaguti, S. Kelner, P. Kent, A. Kimura, T. Kodama, R. Kokoulin, M. Kossov, H. Kurashige, E. Lamanna, T. Lampan, V. Lara, V. Lefebure, F. Lei, M. Liendl, W. Lockman, F. Longo, S. Magni, M. Maire, E. Medernach, K. Minamimoto, P. Mora de Freitas, Y. Morita, K. Murakami, M. Nagamatu, R. Nartallo, P. Nieminen, T. Nishimura, K. Ohtsubo, M. Okamura, S. O’Neale, Y. Oohata, K. Paech, J. Perl, A. Pfeiffer, M. G. Pia, F. Ranjard, A. Rybin, S. Sadilov, E. Di Salvo, G. Santin, T. Sasaki, N. Savvas, Y. Sawada, S. Scherer, S. Sei, V. Sirotenko, D. Smith, N. Starkov, H. Stoecker, J. Sulkimo, M. Takahata, S. Tanaka, E. Tcherniaev, E. Safai Tehrani, M. Tropeano, P. Truscott, H. Uno, L. Urban, P. Urban, M. Verderi, A. Walkden, W. Wander, H. Weber, J. P. Wellisch, T. Wenaus, D. C. Williams, D. Wright, T. Yamada, H. Yoshida, and D. Zschiesche, Nucl. Instrum. Methods Phys. Res. A 506, 250 (2003).

${ }^{27}$ K. Pochwalski, R. Broda, and T. Radoszewski, Int. J. Appl. Radiat. Isot. A 39, 165 (1988). 